Nováček, P.; Roháč, J.; Šimánek, J.; Ripka, P.

Metal Detector Signal Imprints of Detected Objects

IEEE Transactions on Magnetics. 2013, 49(1), 69-72.

\title{
Metal Detector Signal Imprints of Detected Objects
}

\author{
Petr Nováček, Jan Roháč, Jakub Šimánek, and Pavel Ripka \\ Department of Measurement, Faculty of Electrical Engineering, Czech Technical University in Prague, Prague 166 27, Czech \\ Republic
}

\begin{abstract}
Humanitarian demining missions are activities in which an operator safety and time consumption are key issues. To increase a discrimination ability of ATMID metal detector, which we have been using, we extended the capability of the detector with mounting inertial measurement unit (IMU) supplemented by two optical distance sensors on the detector head. That enabled us to perform dead reckoning based on accelerations and angular rates measured by IMU in all three axes. Optical distance sensors have been used for compensation purposes and an initial distance measurement. Our main aim was to interconnect magnetic imprint sensed by the detector with precise localization of its head, which led to imprint size estimation as well as its position. Due to low-cost micro-electro-mechanical system (MEMS) based IMU implementation we have had to deal with unstable dead reckoning outcomes. For this reason we used our designed complex magnetic markers (CMMs) which demarked a searched area plus provided us with precise positioning at its both edges. The main contribution of this paper is in the study and identification of CMM magnetic imprints characteristics and their differences related to various aspects of CMM usage during demining procedure and its conditions. The characteristics of CMMs have been studied and analyzed according to several laboratory experiments and results are presented.
\end{abstract}

Index Terms—Eddy currents, inertial navigation, magnetic field measurement, signal processing.

\section{INTRODUCTION}

$\mathbf{T}$ HIS paper deals with local navigation of a metal detector for detecting and discriminating mines. Precise localization and attitude estimation of the detector in space enables the user to create signal maps, which can be used to increase the discrimination ability of the metal detector [1]. The motivation is to reduce risks for detector operators during humanitarian demining missions [2]. Traditional systems of this type use multisensor arrays and feature extraction image processing methods [3].

Humanitarian demining is still actual problem. Especially in areas of recent military conflict, there is a need to return contaminated land to civilians. This land is needed for agricultural purposes and for other civilian activities, so it must be absolutely free of unexploded ordnance. Absolute land clearance sets high requirements on demining procedures. The problems are greater in areas that are difficult to access. In these areas, handheld mine and metal detectors are the only equipment that can be used [4]. As demining progresses, contaminated areas that are easy to access and demined are the first to be cleared. Other areas that need to be cleared are often handicapped by the presence of buried conductive or metal objects or by characteristics of the soils themselves [5]. Metal contamination and magnetic soils reduce the sensitivity of metal detectors; soil contamination also increases the numbers of false alarms which, in some areas, account for as much as $99.9 \%$ of all alarms [6]. False alarms additionally lower the operator's concentration, and the danger of injuries and casualties arises in combination with reduced detector sensitivity and the operator attention.

A simple system for increasing the discrimination ability of metal detectors needs to be developed in order to reduce humanitarian demining risks. Instead of multisensor arrays such a system should be based on a single metal detector which is manually scanning a small area while its position is monitored with $\mathrm{cm}$ precision. The first attempt towards such a system was reported in [7]. The position of a metal detector was evaluated from inertial navigation system. The integrated errors accumulated over time were unacceptable: up to $1 \mathrm{~m}$ after $90 \mathrm{~s}$ of operation. This was improved by aiding of zero-velocity sensor, but only for motion constrained to a plane. In this paper we suggested to use magnetic markers for position aiding. The advantage of the magnetic markers is that they are sensed directly by the metal detector itself unlike the optical markers which require additional camera [8]. A discrimination ability of metal detectors mainly depends on the level of output signal amplitude and its phase shift which are affected by the conductivity and permeability of a detected object. Thus, these parameters are considered as crucial factors. Furthermore, a lateral and vertical profiles also have to be taken into account [9].

The main contribution of our approach to increase discrimination ability of a metal detector relies on the development of complex magnetic markers (CMMs), for more details see [10]. When they are placed at both edges of searched area and are passed by a detector searching head they provide precise positioning capability as well as their identification, all provided by unique magnetic imprints with a known intensity map. In the case when a database of these intensity maps is built it provides a powerful tool increasing the precision of position evaluation during the demining procedure no matter the conditions. Therefore, we made several CMMs with a different structure and evaluated related reference intensity maps when the searching head was moved with a constant speed and distance above the CMMs. One example of CMM structure is shown in Fig. 1.

The CMMs were formed by the array of plates made from different conductivity materials with a known relative permeability $\mu_{r}$ and conductivity $\sigma$. In our case, the CMM consisted of the combination of aluminum $\left(\mathrm{Al}: \mu_{r \cdot A l}=1, \sigma_{A l}=3.610^{7} \mathrm{~S} / \mathrm{m}\right)$ and chrome steel $\left(\mathrm{St}: \mu_{r S t}=150, \sigma_{S t}=0.4610^{7} \mathrm{~S} / \mathrm{m}\right)$ circular headed nails with the same diameter $r=9.4 \mathrm{~mm}$.

The bottom part under the nails was wooden with the possibility to change nails' structure and distance among them. The 


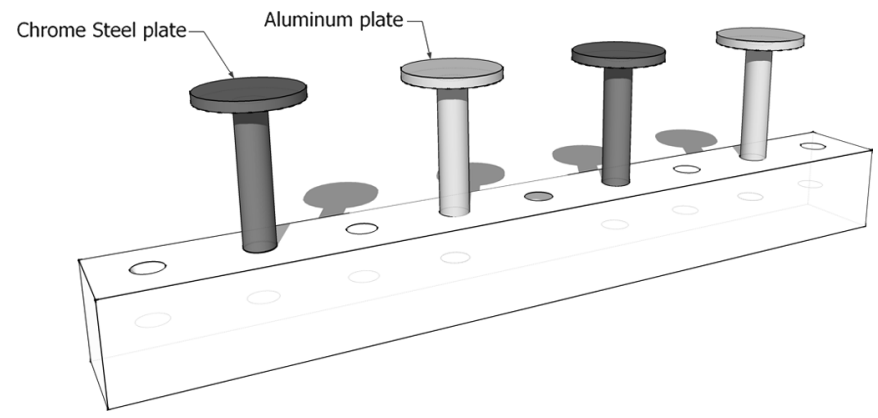

Fig. 1. Sample of the CMM structure with four nails (steel, aluminum, steel, and aluminum) with gaps of $2 \mathrm{~cm}$.

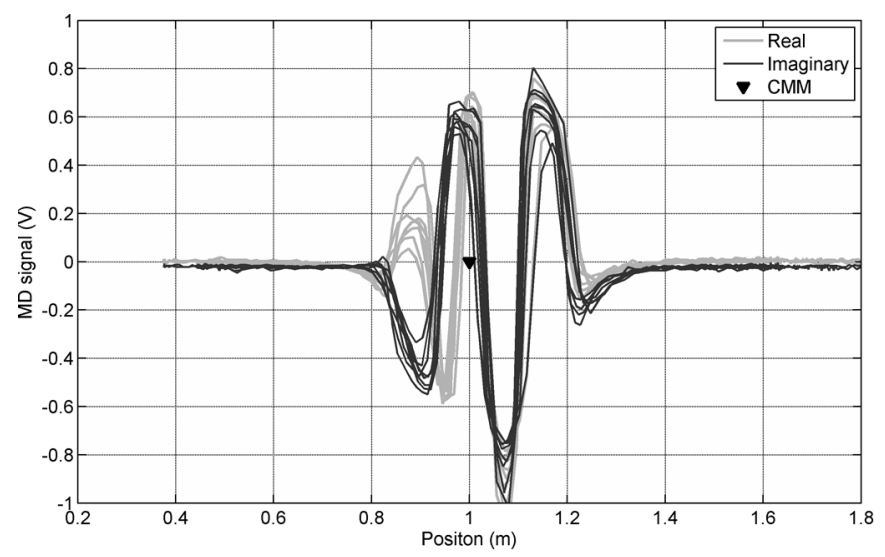

Fig. 2. Imprint of CMM consisting of two nails - steel and aluminum; distance between the nails: $50 \mathrm{~mm}$. [10].

CMM imprints have different character than unexploded ordnance so the CMM and objects being searched can be clearly distinguished. The impact of different materials used in CMMs on the detector output voltage consisted of a real and imaginary part is shown in Fig. 2. When reference imprints are known it is possible to compare them with measured ones, which are influenced by the lateral and vertical distances between the CMM and the searching head and, of course, by the speed, and attitude of the head. All these aspects have been studied and characterized by performed experiments and results are presented.

\section{Measurement Setup}

All data and results presented were obtained under laboratory conditions. Our experiment setup consisted of ATMID [11] metal detector supplemented by additional information sources such as inertial measurement unit (IMU) for accelerations and angular rates sensing, plus two infrared distance sensors, and complex magnetic markers (CMMs). Usage of ground distance sensors is a second major improvement of the system described in [8].

For data validation purposes we placed a camera below the laboratory ceiling which provided us with the position and velocity reference to relate dead-reckoning outcomes to. The reference position estimation has had a sub pixel resolution and approximately $1 \mathrm{~mm}$ standard deviation, which guaranteed required precision for the CMM characteristics study.

All data measured by the detector itself, IMU, and distance sensors were synchronized by an acquisition unit and sent to

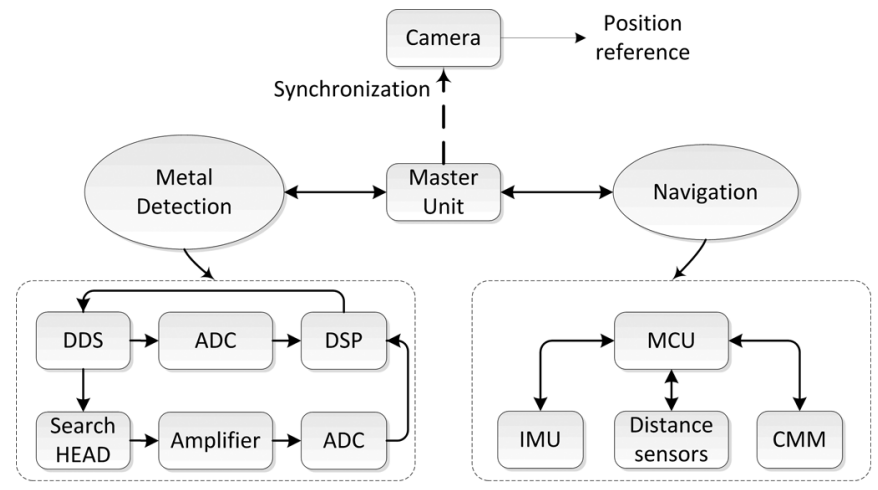

Fig. 3. Principle scheme of the measurement setup. A/D converter (ADC), digital signal processor (DSP), direct digital synthesizer (DDS), inertial measurement unit (IMU), micro-controller unit (MCU), complex magnetic marker (CMM).

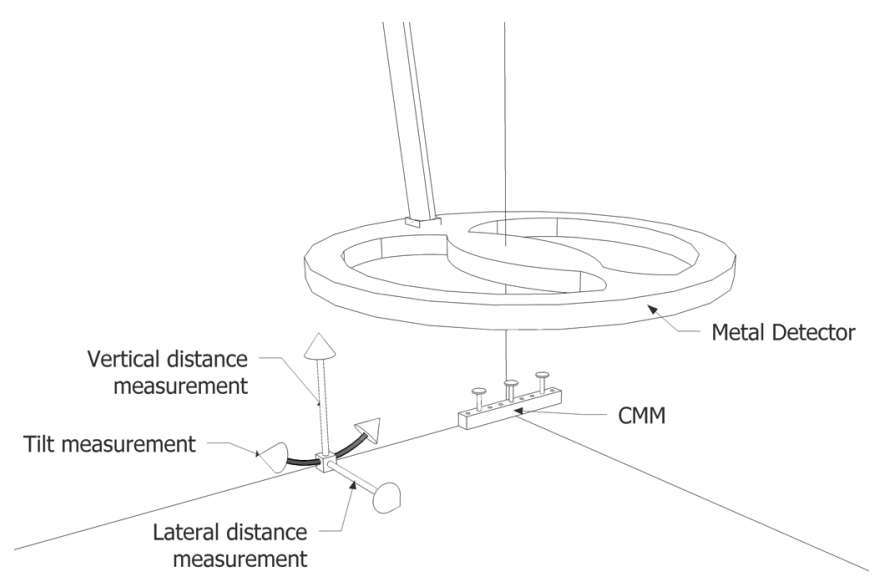

Fig. 4. Measurement setup and procedure.

a PC via communication interface. The sampling frequency of these data was $50 \mathrm{~Hz}$. A principal schema is shown in Fig. 3.

For the CMM characteristics study the experiments observed only the detector output signals representing CMM imprints and the detector position obtained from the reference position camera system [12]. The reference position system was not synchronized with the other data; it is future possibility for the system capability extension; however, its update rate corresponded also to 50 frames per second.

\section{EXPERIMENTAL RESULTS}

The experiments studying different aspects of CMM magnetic imprints characteristics were performed with more compositions of CMMs. The first one was formed by only aluminum nails and the other one consisted of a combination of aluminum and chrome steel nails. Nevertheless, results presented in this paper were related just to CMM formed by three aluminum plates (as shown in Fig. 4), because the dependencies were very similar.

\section{A. Observation of the Detector Sensitivity}

Evaluation of a metal detector output signal, when a small sphere with $10 \mathrm{~mm}$ diameter was observed, led to intensity map shown in Fig. 5. The intensity map was constructed by digitizing real and imaginary parts of detector output signal and then 


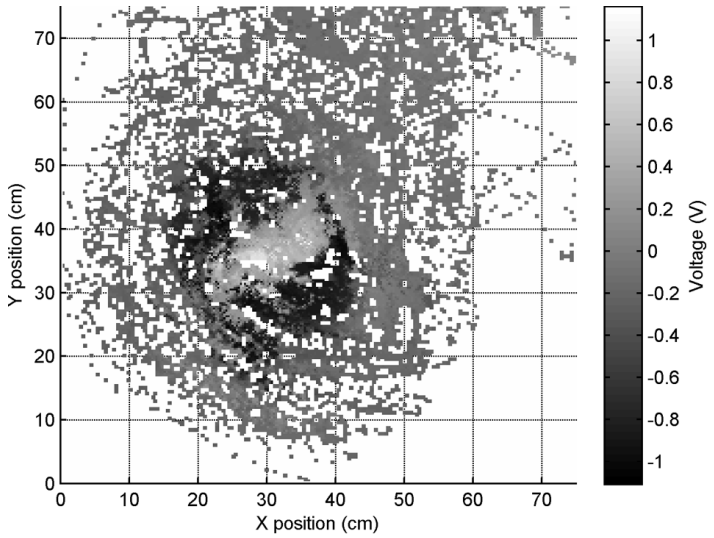

Fig. 5. Imprint intensity map measured by ATMID metal detector with INOX AISI 420 sphere of diameter $10 \mathrm{~mm}$ as the detected target.
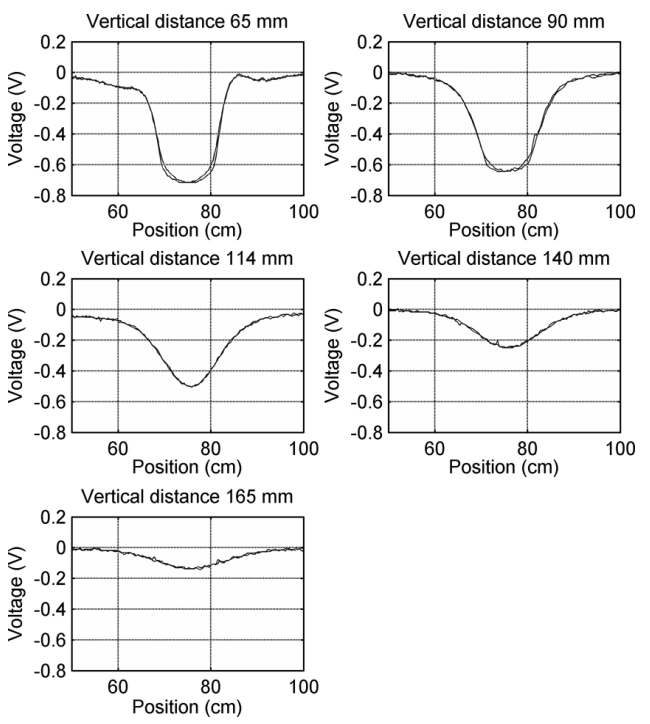

Fig. 6. Imprint dependency on a vertical distance between CMM and the detector head.

converted into voltage magnitude and corresponding phase. In Fig. 5 there is shown the evaluated magnitude with respect to the position and distance of the sphere from the detector head. A color representation corresponds to the value of the magnitude.

\section{B. CMM Imprint Dependency on Lateral and Vertical Profile}

Due to the fact that a detector operator does not need to pass the CMM precisely and the application still requires precise CMM identification we performed several experiments to study the imprint intensity map dependency on CMM vertical distance from the detector head as well as its dependency on CMM lateral distance from a detector main axis of sensitivity. Imprint parameters dependency on a vertical distance between CMM and the detector head is shown in Fig. 6. The detector head was moved above the CMM with different vertical distances $(65,90$, 114,140 , and $165 \mathrm{~mm}$ ). The values were chosen with respect to the ATMID detector sensitivity.

A similar character of the imprint parameters was also observed in the case of a lateral distance dependency. This dis-
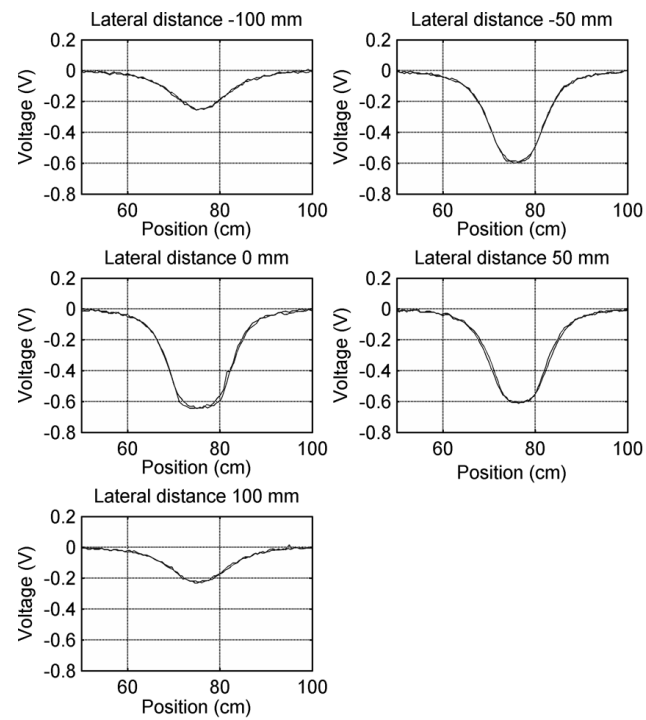

Fig. 7. Imprint dependency on a lateral distance between CMM axis and the detector main axis.

TABLE I

IMPRINTS AMPLITUDE DEPENDENCIES

\begin{tabular}{|c|c|c|c|}
\hline $\begin{array}{c}\text { Vertical } \\
\text { Distance }\end{array}$ & Amplitude & $\begin{array}{c}\text { Lateral } \\
\text { Distance }\end{array}$ & Amplitude \\
\hline $65 \mathrm{~mm}$ & $0.72 \mathrm{~V}$ & $-100 \mathrm{~mm}$ & $0.25 \mathrm{~V}$ \\
\hline $90 \mathrm{~mm}$ & $0.65 \mathrm{~V}$ & $-50 \mathrm{~mm}$ & $0.60 \mathrm{~V}$ \\
\hline $114 \mathrm{~mm}$ & $0.51 \mathrm{~V}$ & $0 \mathrm{~mm}$ & $0.65 \mathrm{~V}$ \\
\hline $140 \mathrm{~mm}$ & $0.25 \mathrm{~V}$ & $50 \mathrm{~mm}$ & $0.61 \mathrm{~V}$ \\
\hline $165 \mathrm{~mm}$ & $0.13 \mathrm{~V}$ & $100 \mathrm{~mm}$ & $0.23 \mathrm{~V}$ \\
\hline
\end{tabular}

Comparison of signal amplitudes related to specific vertical and lateral distances of CMM axis from the detector head main axis.

tance was measured between the detector main axis and the axis of the CMM. The character is shown in Fig. 7. The detector head was moved above the CMM at the vertical distance $90 \mathrm{~mm}$ and lateral distances varied $(-100,-50,0,50$, and 100 $\mathrm{mm})$. The distances were chosen with respect to the detector head diameter and its middle axis, which was kept constant, and the CMM position varied. The diameter of the ATMID detector head is $280 \mathrm{~mm}$.

The results from experiments studying the imprint parameters dependency on CMM vertical and lateral distances from the detector head are summarized in Table I, in which maximal amplitudes of the detector output signal are provided.

\section{CMM Imprint Dependency on the Detector Head Attitude}

Because there is not possible generally to ensure null attitude of the detector head with respect to CMM origins, we also analyzed the impact of different attitude on the CMM imprint characteristics. We observed the amplitude of the imprint and the behavior did not show significant changes in the attitude range of $\pm 20^{\circ}$. The resulting imprints for different attitude are shown in Fig. 8.

\section{CONCLUSION}

This paper deals with CMMs and their magnetic imprints. The main contribution of this paper is in analyzing imprint char- 

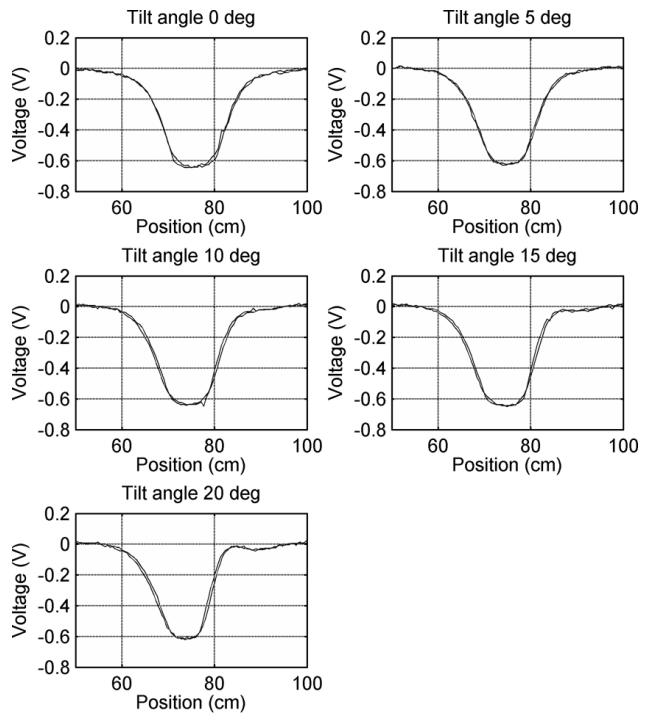

Fig. 8. CMM imprint dependency on a detector head attitude- - tilt angle set in the range from $0^{\circ}$ up to $+20^{\circ}$ with the step of $5^{\circ}$.

acteristics dependency on various conditions of a metal detector handling. Precise knowledge of CMMs position plays a crucial role in dead reckoning process in which the actual position of the detector head is estimated. CMMs position is used to compensate position errors mainly caused by uncompensated biased data measured by MEMS accelerometers and angular rate sensors. To localize CMMs it is required to identify CMMs correctly. The identification relies on correct matching of the measured CMM imprint with the reference one saved in the system memory. For the matching it is needed to know how the imprint can be affected by an operator handling the detector. During a demining procedure vertical and lateral distances between CMM and the detector head, and the detector head attitude can vary when CMM is passed by the detector head. Therefore, in this paper we analyzed all these aspects on two different CMMs. Even if all experiments were performed under laboratory conditions, we can clearly specify changes of CMM imprints according to mentioned handling conditions. Experiments proved the imprint amplitude was changing with lateral and vertical distances defined between CMM and the detector head. In ranges calculation we considered the environmental impact on the imprint on which it caused maximal amplitude perturbation of $0.1 \mathrm{~V}$. Thus, to ensure correct identification we increased the minimal amplitude level for the CMM identification to be $0.2 \mathrm{~V}$. In this case the correct identification as well as position determination can be done in ranges up to $105 \mathrm{~mm}$ in vertical distance and up to $90 \mathrm{~mm}$ in lateral distance. Both values were calculated based on Table I and progress approximations. Based on other experiments analyzing attitude changes impact on CMM identification it was proved that this aspect had no significant importance for its observation, because according to two different CMMs application we did not recognize any influence of different attitude in range of \pm 20 deg on their magnetic imprints.

When CMM imprints are identified and their position precisely evaluated dead reckoning process can be strongly improved in the position estimation in the whole range of the metal detector application. It further increases discrimination ability of metal detectors during humanitarian demining missions as well as it decreases injury risks of detector operators because of better ability in differentiation among metal scraps and dangerous objects such as explosive remnants of wars.

\section{ACKNOWLEDGMENT}

This work was supported in part by the research program GD102/09/H082, "Sensors and Intelligent Sensor Systems," at CTU, Prague, sponsored by the Czech Science Foundation; and by the Grant Agency of the Czech Technical University, Prague, under Grant SGS10/288/OHK3/3T/13, "Modular system for attitude and position estimation."

\section{REFERENCES}

[1] A. Brancaccio and G. Leone, "Localization of buried objects," in Proc. 13th Int. Conf. Ground Penetrating Radar (GPR), 2010, pp. 1-4.

[2] H. Kruger and H. Ewald, "Handheld metal detector with online visualisation and classification for the humanitarian mine clearance," IEEE Sens., 2008

[3] M. D. Tran, C. Abeynayake, and L. C. Jain, "A target discrimination methodology utilizing wavelet-based and morphological feature extraction with metal detector array data," IEEE Trans. Geosci. Remote Sens., vol. 50, no. 1, pp. 119-129, Jan. 2012.

[4] G. Kellermann, "Metal detector, in particular mine detector," US Patent 726555,2007

[5] P. Ripka, A. M. Lewis, and J. Kubik, "Mine detection in magnetic soils," Sens. Lett., vol. 5, no. 1, pp. 15-18, 2007.

[6] A. Lewis, T. Bloodworth, D. Guelle, and A. Smith, Metal-Detector Handbook for Humanitarian Demining 2003 [Online]. Available: http://publications.jrc.ec.europa.eu/repository/handle/111111111/ 13314

[7] B. Barrow and N. Khadr, "A combined EMI sensor and inertial motion system for localized UXO interrogation," presented at the UXO COUNTERMINE RANGE Forum, Las Vegas, NV, 2006.

[8] X. Feng, M. Sato, and C. Liu, "Subsurface imaging using a handheld GPR MD system," IEEE Geosci. Remote Sens. Lett., vol. 9, no. 4, pp. 659-662, 2012.

[9] C. Bruschini, "A multidisciplinary analysis of frequency domain metal detectors for humanitarian demining," Ph.D. dissertation, Faculty Appl. Sci., Vrije Univ., Brussels, Belgium, 2002.

[10] P. Novacek, J. Rohac, and P. Ripka, "Complex markers for a mine detector," IEEE Trans. Magn., vol. 48, no. 4, pp. 1489-1492, Apr. 2012.

[11] "ATMID all Terrain Mine Detector Maintenance Manual MT5001/16/ 010E," Schiebel, Vienna, Austria, 2003, pp. 7-13.

[12] P. Novacek, P. Ripka, O. Pribula, and J. Fischer, "Mine detector with discrimination ability," JEE, vol. 61, no. 7, pp. 141-143, 2010. 